\title{
Single osteocyte gene expression in an in vivo model for load-induced bone adaptation
}

(1)

\author{
Robin Wilson ${ }^{1}$, Andreas Trüssel1, Duncan Webster ${ }^{1}$, Felix Kurth ${ }^{2}$, Petra Dittrich ${ }^{2}$, Raljph Mülller
}

${ }^{1}$ Institute for Biomechanics, ${ }^{2}$ Bioanalytics Group, EJH Zürrich, Zürrich, Switzerland

Introduction

Methods

Results

Conclusions

References

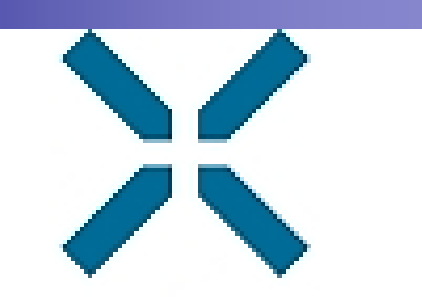

It is hypothesized that osteocytes regulate bone adaptation by sensing mechanical strains in their microenvironments and signaling net bone formation or resorption. ${ }^{1}$ Due to bone's anisotropic architecture, mechanical strain has high spatial variability on the microscopic scale. It follows that bone adaptation exhibits a similar variability. Thus, it is crucial to analyze small volumes (1-10 cells) and maintain spatial information. Our aim is to accurately determine the relationships between mechanical strain, osteocyte behavior, and load-induced bone remodeling by spatially linking three models of a single vertebra: i) micro-finite element model for mechanical strain ${ }^{2}$ ii) bone adaptation using 3D registration of time-lapsed $\mu \mathrm{CT}^{3}$ iii) ex vivo osteocyte gene expression by qRT-PCR of single cells or small cell populations. In this work, we optimized methods for osteocyte gene expression analysis including tissue preparation, laser microdissection, and GRT-PCR. This strategy allows for very high spatial resolution of osteocyte gene expression in ex vivo tissue, making it possible to examine the relationships between bone adaptation, mechanical strain, and gene expression on a microscopic level. This ability will provide further insight into the cellular mechanisms for load-induced bone adaptation.

Female C57BL/6 mice vertebrae were cryosectioned $(12 \mu \mathrm{m}$ thickness) and dehydrated in an alcohol gradient (ethanol or methanol), with a staining step in $10 \mu \mathrm{g} / \mathrm{mL}$ Hoechst (Hoechst 33342, Sigma) in 75\% alcohol (figure 1A). Sections were microdissected using a P.A.L.M. laser microscope (Zeiss) into PCR tube caps filled with $10 \mu \mathrm{L}$ RT mix (figure 1B). A two-step Taqman qRT-PCR protocol was used for gene expression analysis. Lysis, reverse transcription, and 15 preamplification cycles were performed using the CellsDirect ${ }^{\mathrm{TM}}$ One-Step qRT-PCR kit (Invitrogen). The resulting CDNA was diluted and analyzed using a standard Taqman qPCR protocol. Samples were evaluated for osteocyte markers (SOST and DMP1), an osteoblast marker (ALPL), and a housekeeping gene (HPRT1). Using this protocol, we first determined sample stability over time since RNA is prone to degradation. Over two hours, multiple samples ( 10 cells each) were microdissected from the same section at discrete time intervals (figure 2) and directly reverse transcribed. Sample stability was analyzed by the percentage of detectable signals $(23<\mathrm{Ct}<28)$ in each time window. We then tested our ability to selectively analyze specific tissue by comparing gene expression of osteocytes and bone marrow cells ( $\sim 10$ cells).

In our study of sample stability over time, samples dehydrated in ethanol showed a loss of reliable signal after 20 min in ambient conditions, while populations dehydrated in methanol did not show a decreased signal after 120min (figure 2). Therefore, methanol dehydration was used in all following studies. Next, gene expressions of specific tissue samples were analyzed. Expressions of SOST, DMP1, and ALPL were analyzed relative to the expression of a housekeeping gene, HPRT1 (figure 3). As expected, osteocytes expressed significantly higher levels of both osteocyte markers, SOST and DMP1 $(p<0.05)$

We have developed a method for microdissection and gene expression analysis of small numbers of ex vivo cells. This high specificity will allow us to map gene expression back into the CT volume in the future. With this information, we can correlate gene expression with local bone adaptation and local strain, thus giving us insight into the role of osteocytes as mechanosensors.

1. Bonewald. 2011. J Bone Miner Res. 26.

2. Webster et al. 2008 CMBBE 11

3. Schulte et al. 2011. Bone 48.

This project is funded by SystemsX, the Swiss initiative in systems biology. We declare no conflicts of interest.

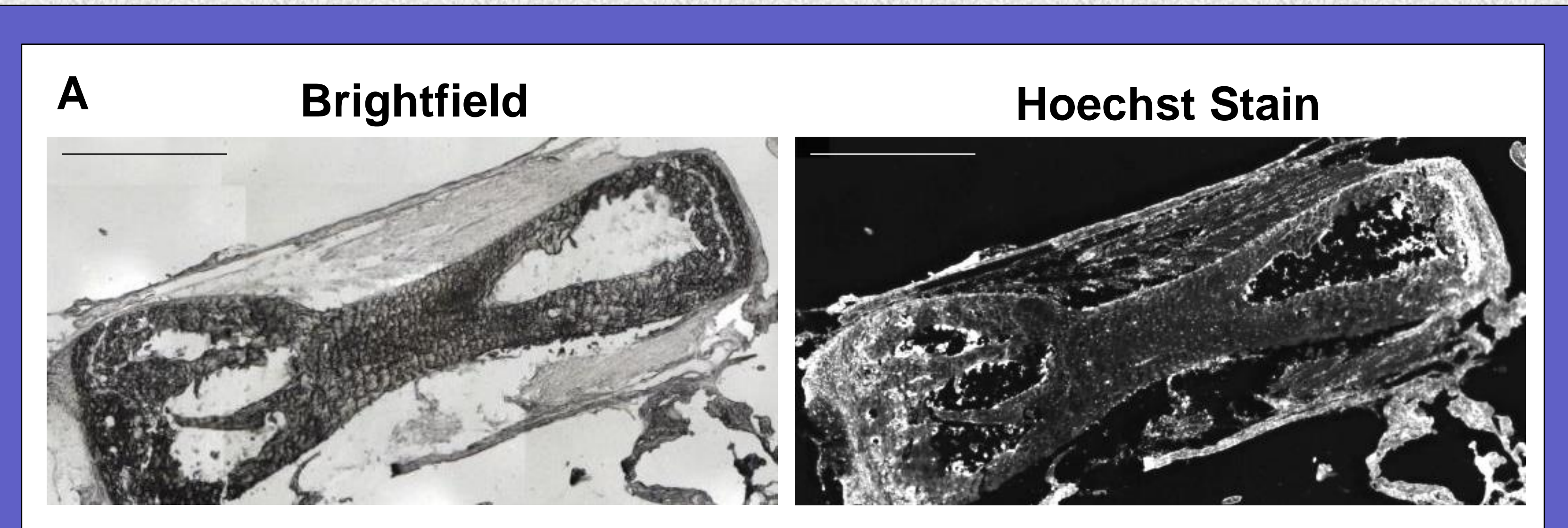

B
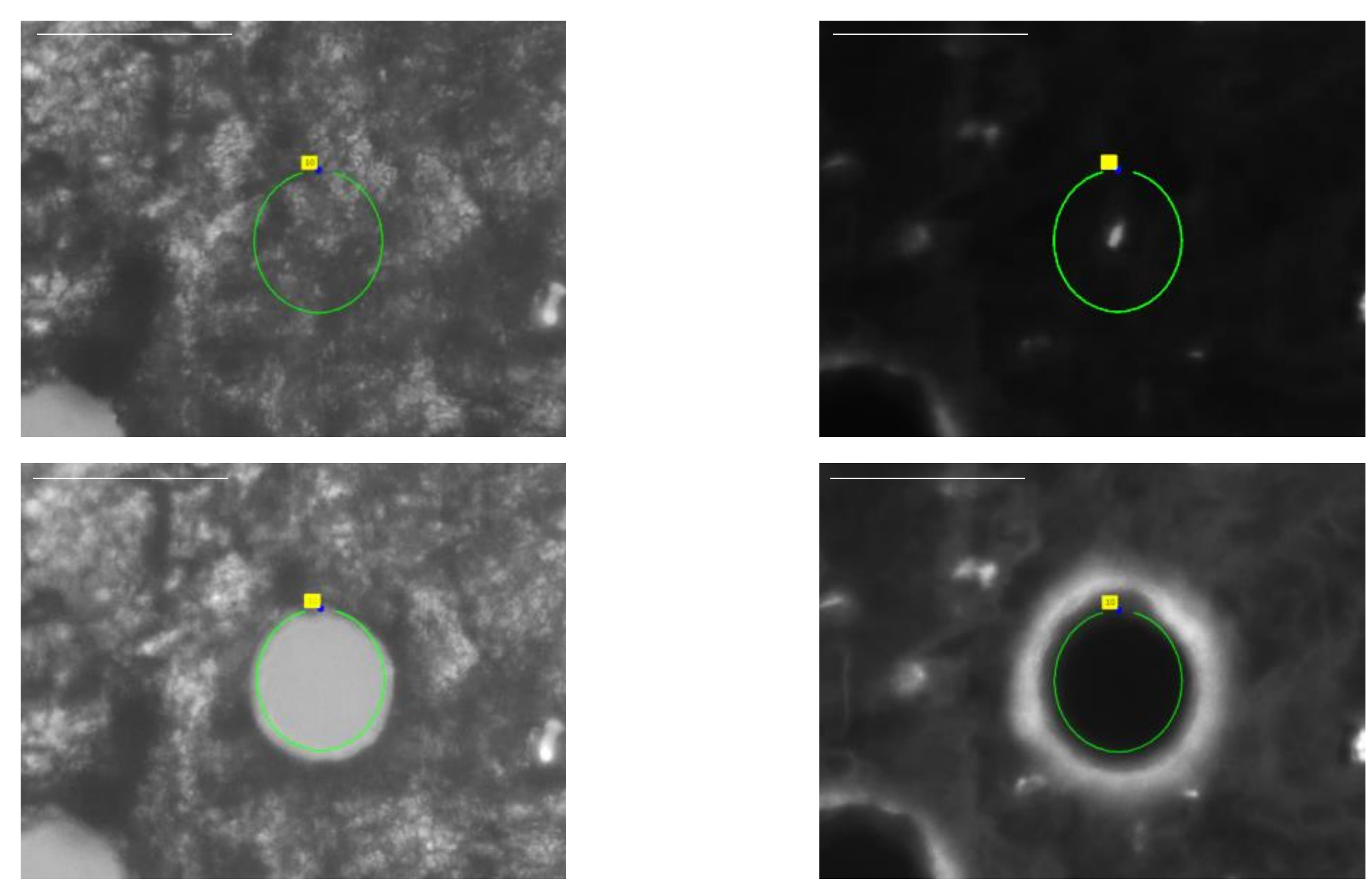

Figure 1: Calcified C57BL/6 mouse vertebra cryosection after methano dehydration with Hoechst staining (A). Images of a single osteocyte in the bone matrix before and after microdissection. Hoechst stain was used to identify embedded osteocytes (B). Scale bars: $1 \mathrm{~mm}(\mathrm{~A}) 50 \mu \mathrm{m}(\mathrm{B})$

Time stability of microdissected osteocyte qPCR signal

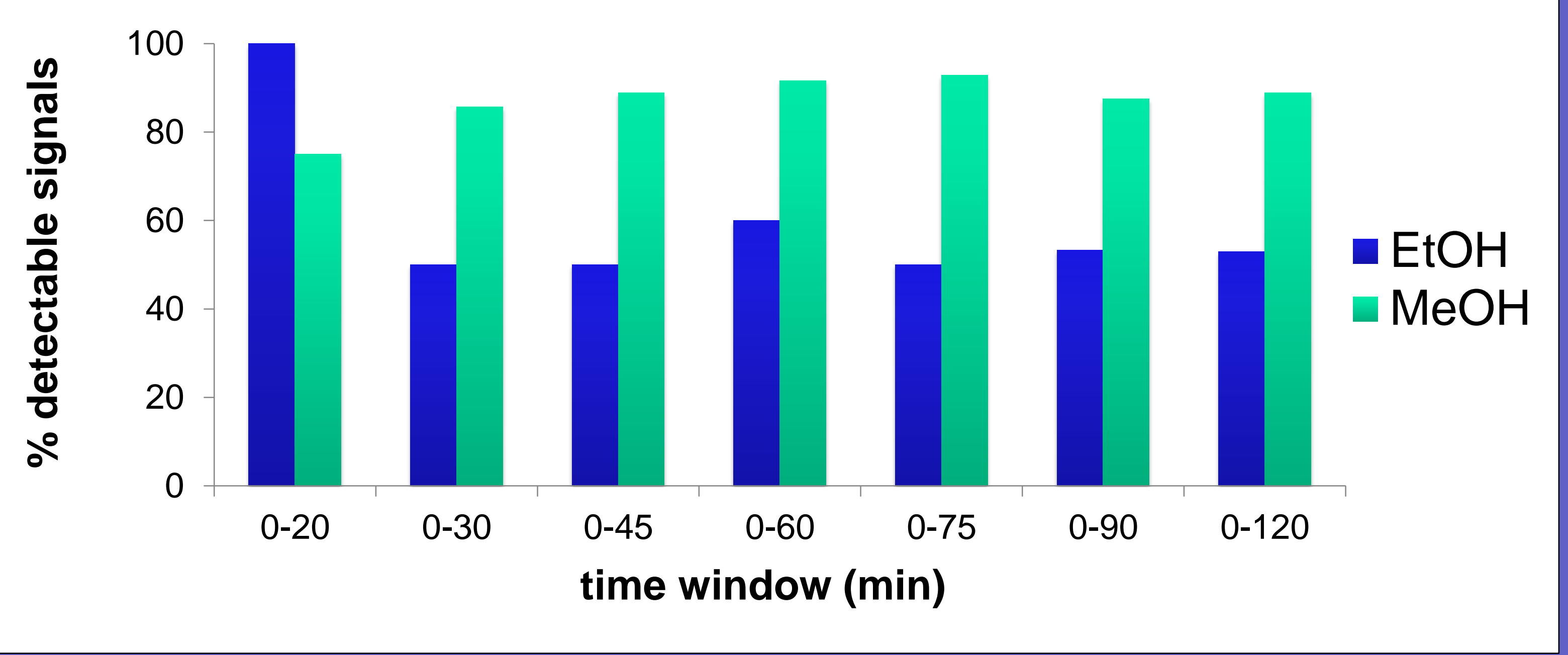

Figure 2: Approximately 10 cells were microdissected for each qPCR sample and analyzed for HPRT1 expression over time. In each tria $(n>3)$, samples were harvested from the same bone section over a period of two hours in order to determine the time stability of RNA. Sections were dehydrated in an ethanol or methanol gradient. The plot shows the percent detectable signals for the indicated time interval between dehydration and reverse transcription. These results show that methanol dehydration is superior to ethanol dehydration for sample stability over time.

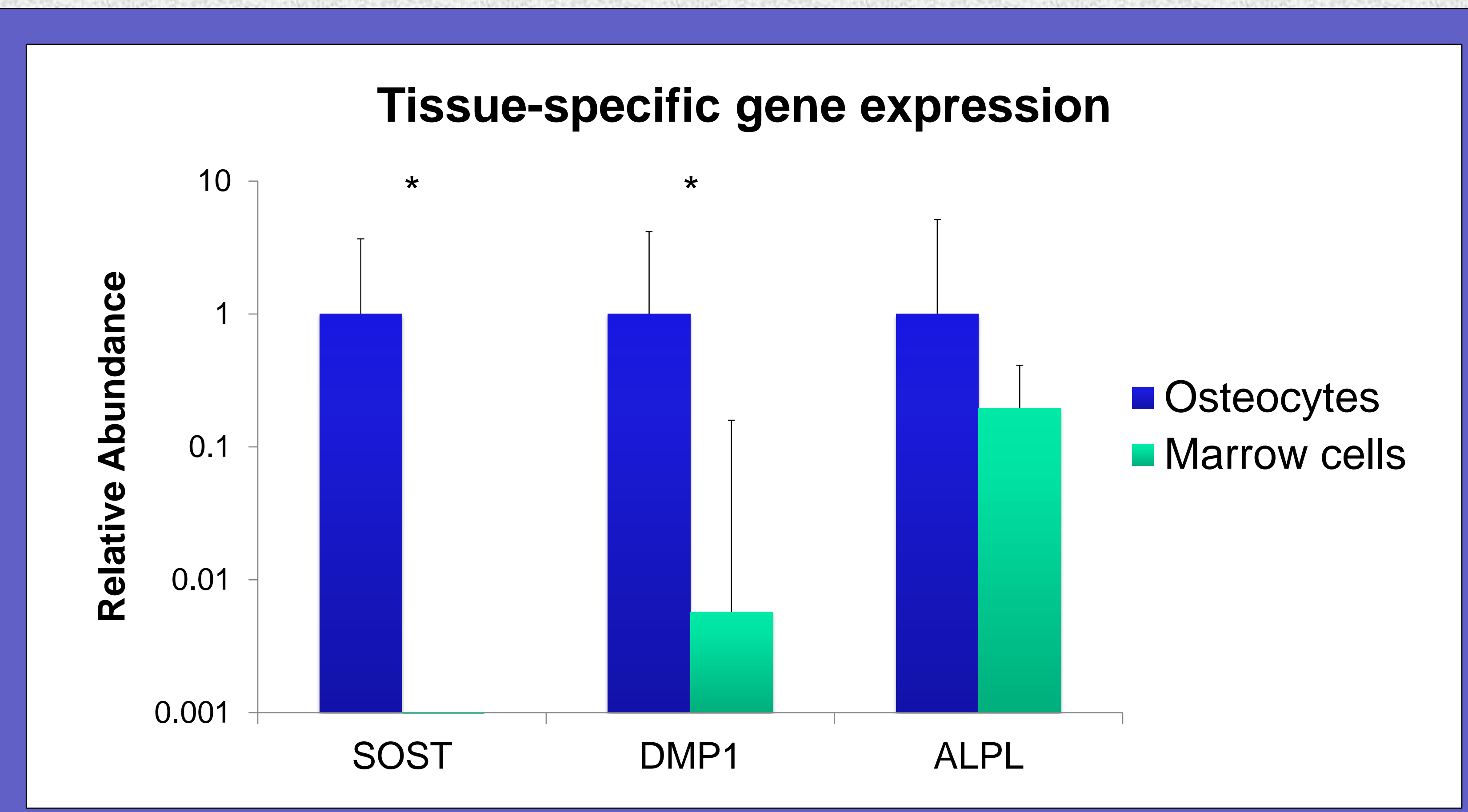

Figure 3: qPCR results for tissue-specific samples normalized to the housekeeping gene, HPRT1 ( ${ }^{*}$ indicates $\left.p<0.05\right)(n=3)$. Both SOST and DMP1 are osteocyte markers, while ALPL is an osteoblast marker. ${ }^{1}$ Our results show that osteocytes expressed $>150,000 x$ SOST, 175x DMP1, and $5 x$ ALPL compared to marrow cells. These results indicate that we were able to select and microdissect specific tissue within bone. 